\title{
PRIMER REGISTRO DEL GÉNERO STROMATONEUROSPORA (ASCOMYCETES, XYLARIACEAE) EN MÉXICO
}

\author{
Rosario Medel \\ Instituto de Ecología, A.C. \\ Apartado postal 63 \\ 91000 Xalapa, Veracruz
}

\section{RESUMEN}

Se registra por primera vez en México a Stromatoneurospora phoenix, un hongo perteneciente a la familia Xylariaceae, encontrado en el estado de Yucatán.

Palabras clave: Stromatoneurospora phoenix, Ascomycetes, México.

\begin{abstract}
Stromatoneurospora phoenix a fungus belonging to the Xylariaceae is recorded for the first time for Mexico. It was collected in the state of Yucatan.

Key words: Stromatoneurospora phoenix, Ascomycetes, Mexico.

\section{INTRODUCCIÓN}

Dentro de los Ascomycetes, la familia Xylariaceae es la mejor representada en México, pues cuenta con más de 240 especies registradas (Medel et al., 1999). San Martín et al. (1998) enlistan para el país 24 géneros, aunque no mencionan al género Hypocopra (Fr.) J. Kickx, el cual fue citado de varias entidades de México por Krug y Cain (1974).

Como parte de los estudios relacionados con los ascomicetos mexicanos, se presenta en este trabajo el primer registro de Stromatoneurospora Jong \& Davis, un género singular de Xylariaceae, cuyas dos especies aceptadas crecen principalmente en suelos o sustratos afectados por recientes fuegos, especialmente sobre tallos u otros restos de gramíneas (Dennis, 1957, 1958; Jong y Davis, 1973; Rogers et al., 1992).

El material estudiado fue examinado siguiendo las técnicas de rutina en micología, empleando preparaciones en $\mathrm{KOH}$ a $10 \%$, solución de Melzer y floxina. El espécimen revisado se encuentra depositado en la colección de hongos del herbario del Instituto de Ecología, A.C. (XAL).
\end{abstract}


Stromatoneurospora phoenix (Kunze) Jong \& Davis, Mycologia 65: 459. 1973. Figs. 1-6.

$=$ Chromocreopsis striispora Stevenson

= Hypoxylon phoenix (Kunze) Berk. \& M.A. Curtis

= Neurospora phoenix (Kunze) Dennis

= Sarcoxylon phoenix (Kunze) Petrak

= Sphaeria phoenix (Kunze)

= Xylaria phoenix (Kunze) Dennis

= Xylosphaera phoenix (Kunze) Dennis

Estroma solitario o gregario, de 1.5-3 mm de diámetro, pseudoestipitado o sésil, en ocasiones sólo se observan los peritecios solitarios esparcidos en el sustrato. Estroma amarillento-naranja, café-anaranjado a de color paja en seco, con la parte interna blanquecina a amarillenta pálida. Estroma con los ostiolos negros. Peritecios globosos a subglobosos, pequeños, menores de $0.1 \mathrm{~mm}$ de diámetro, con ostiolos mamiformes.

Ascas estipitadas, de 96-120 x 8-8.8 (-9.6) $\mu \mathrm{m}$ en la parte que contiene las esporas, con estípite de 32-40 $\mu \mathrm{m}$ de longitud, octosporadas, amiloides. Poro ascal en forma de sombrero invertido, aplanado, la parte más ancha (parte superior) de 4.8-5.6 $\mu \mathrm{m}$ de diámetro por $1.6 \mu \mathrm{m}$ de ancho, el poro ascal en vista superior se observa de forma anular. Ascosporas elípticas a elíptico-fusiformes, de 16-18.4 (-20) x 8-8.8 $\mu \mathrm{m}$, hialinas cuando jóvenes, a amarillentas 0 de color café obscuro cuando maduras, ornamentadas con estrías longitudinales completas e incompletas, las estriaciones son notorias incluso en ascosporas jóvenes, sin poro ni línea germinal. Perisporio delgado, no dehiscente en $\mathrm{KOH}$ a $10 \%$. Parafisas no se observaron.

Material estudiado: Yucatán, Dzibilchaltún, zacatal, pastizal H. Cemé 98, 239 (XAL).

Discusión: El material examinado concuerda con las descripciones de Jong y Davis (1973) y Dennis $(1957,1958)$. Dennis (1958) registró esporas de 7-9 $\mu \mathrm{m}$ de ancho, lo cual concuerda con el material revisado, no así con Jong y Davis (1973) quienes las observaron más estrechas $(6-8 \mu \mathrm{m})$.

Stromatoneurospora phoenix, es un hongo muy peculiar que exhibe características de por lo menos tres familias diferentes: de Hypocreaceae por el estroma y color brillante, de Sordariaceae las esporas estriadas, y de Xylariaceae por el poro ascal apical y las paráfisas basales (Jong y Davis, 1973).

El material estudiado se localizó creciendo en pastos quemados. De acuerdo con Rogers et al. (1992), es muy verosímil que la asociación con vegetales incinerados sea de gran importancia para estos hongos, disparándose probablemente un estímulo para la germinación de las ascoporas. A tal hecho se le atribuye particular peso taxonómico.

Con este nuevo registro, suman ya 26 los géneros pertenecientes al familia Xylariaceae que se han encontrado en el país, hecho que la confirma como la más diversa dentro de los Ascomycetes.

La especie fue citada de regiones tropicales de Puerto Rico, Surinam, Venezuela (Jong y Davis, 1973), Uganda, India, Birmania, Filipinas (Dennis, 1957, 1958) y Hawai (Rogers et al., 1992). 

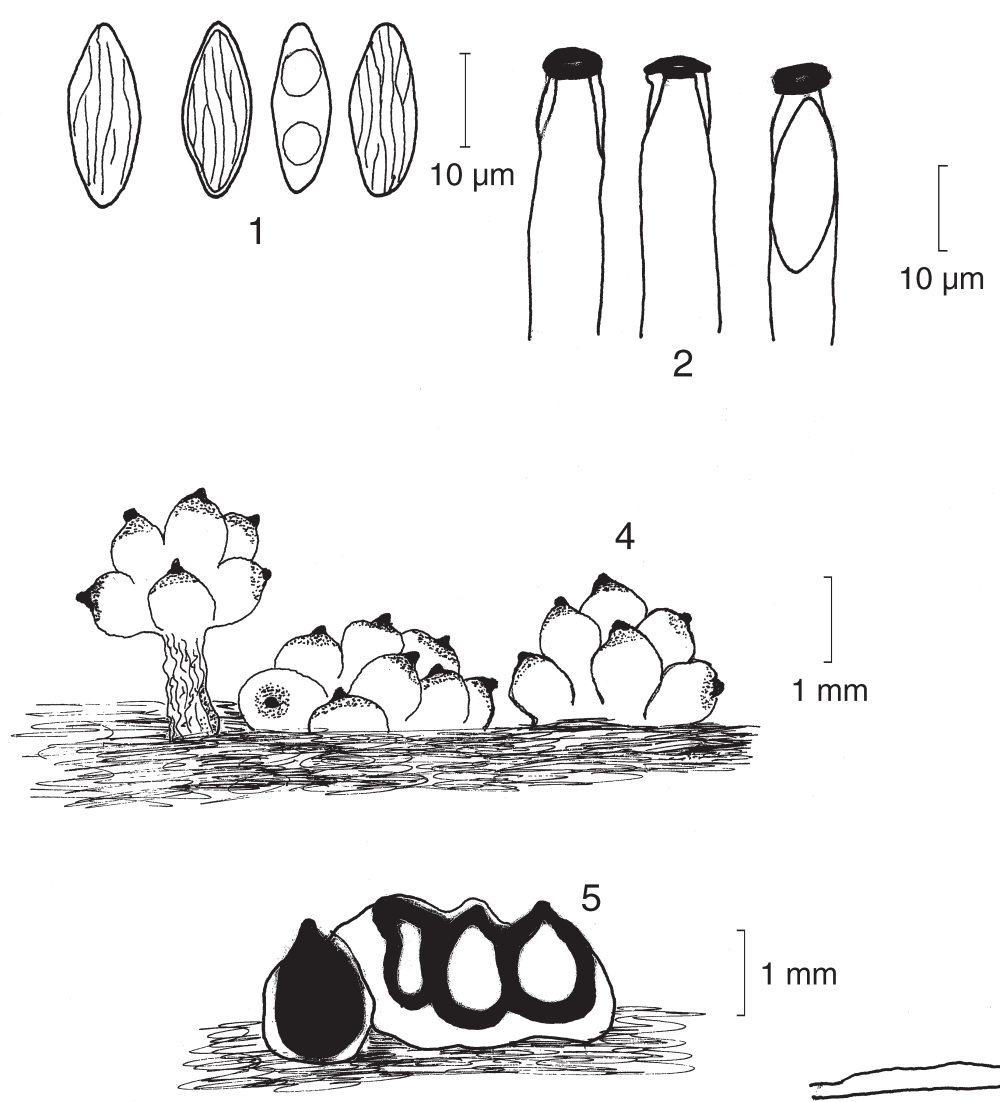

$1 \mathrm{~mm}$

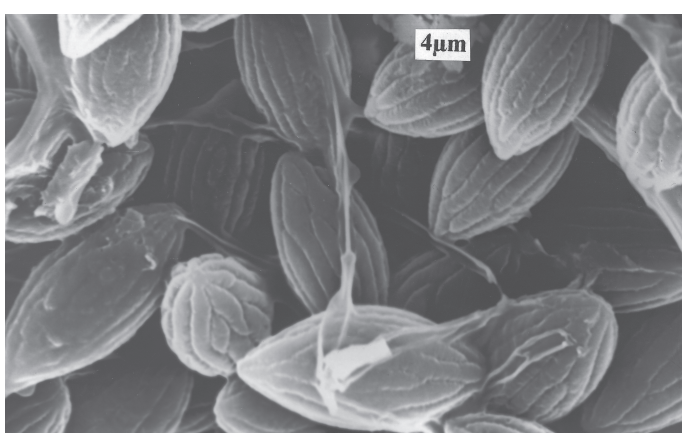

Figs. 1-6. Stromatoneurospora phoenix. 1. Ascosporas; 2. Detalle del poro ascal; 3. Asca con ascosporas; 4. Estromas; 5. Corte transversal del estroma; 6. Fotografía al microscopio electrónico, donde se observan las estriaciones en la superficie de las ascosporas. 


\section{AGRADECIMIENTOS}

La autora agradece al Dr. Francisco Lorea, del Instituto de Ecología, A.C., la revisión del manuscrito. A Juan Lara Carmona y Ma. Eugenia Ramírez, de la misma institución, su apoyo en diversas tareas. Al técnico Tiburcio Laez se le reconoce la toma de fotografías al microscopio electrónico. Al Dr. J. D. Rogers se dan las gracias por su ayuda en la consulta bibliográfica y al Dr. Felipe San Martín por la aportación de información relacionada con el hongo estudiado.

\section{LITERATURA CITADA}

Dennis, R. W. G. 1957. Further notes on tropical American Xylariaceae. Kew Bull. 12: 297-332. Dennis, R. W. G. 1958. Some Xylosphaeras of tropical Africa. Rev. Biol. Lisboa 1: 175-207. Jong, S. C. y E. E. Davis. 1973. Stromatic neurosporas. Mycologia 65: 458-464.

Krug, J. C. y R. F. Cain. 1974. New species of Hypocopra (Xylariaceae). Can. Jour. Bot. 52: 809-843. Medel, R., G. Guzmán y S. Chacón. 1999. Especies de macromicetos citadas de México IX. Ascomycetes parte III: 1983-1996. Acta Bot. Mex. 46: 57-72.

Rogers, J. D., Y.-M. Ju y D. E. Hemmes. 1992. Hypoxylon rectangulosporum sp. nov., and comments on taxa of Podosordaria and Stromatoneurospora. Mycologia 84: 157-165.

San Martín, F., J. D. Rogers y Y.-M. Ju. 1998. Clave dicotómica para los géneros de la familia Xylariaceae (Pyrenomycetes, Sphaeriales) de México. Acta Bot. Mex. 42: 35-41. 